\title{
Sergliflozin Etabonate
}

National Cancer Institute

\section{Source}

National Cancer Institute. Sergliflozin Etabonate. NCI Thesaurus. Code C78129.

A benzylphenol glucoside and selective sodium-glucose co-transporter subtype 2

(SGLT2) inhibitor with antihyperglycemic activity. Its prodrug form, serg liflozin etabonate,

is orally available and is converted to sergiflozin upon absorption. 